\title{
Comparison of photoreceptor outer segment length in diabetic and idiopathic epiretinal membranes
}

\begin{abstract}
Purpose Idiopathic and diabetic epiretinal membranes (ERMs) differ significantly in terms of macular morphology. Our aim was to compare the photoreceptor outer segment (PROS) length in diabetic and idiopathic ERMs before and after surgery. Methods Fifty-two eyes of 50 patients who underwent pars plana vitrectomy (PPV) due to idiopathic and diabetic ERMs were included in this retrospective case series. All of the patients underwent ERM and internal limiting membrane (ILM) removal. The patients had been followed up 6 months postoperatively. Spectral-domain optical coherence tomography (OCT) was used to measure the PROS length, overall PR length and central foveal thickness (CFT).
\end{abstract}

${ }^{1}$ Ophthalmology Department, Beyoglu Eye Training and Research Hospital, Eye Clinic, Istanbul, Turkey

${ }^{2}$ Besni State Hospital, Eye Clinic, Adıyaman, Turkey

${ }^{3}$ Ophthalmology

Department, Pamukkale University, Denizli, Turkey

Correspondence:

G Pekel, Ophthalmology Department, Pamukkale University Medical School, Kınıklı, Denizli, 20070,

Turkey

Tel: +90 2584440728 5785;

Fax: +90 2582134922

E-mail: gkhanpekel@yahoo. com

Received: 21 March 2015 Accepted in revised form: 10 June 2015

Published online:

17 July 2015
Results The PROS length, overall PR length, and CFT were statistically significantly lower at all the postoperative visits when compared to preoperative values in both the idiopathic and diabetic ERM groups $(P<0.001)$. The amount of decrease in PROS and overall PR length were similar in both groups after surgery $(P>0.05)$. There was no relation between amount of PROS length change and visual acuity gain in both groups $(P>0.05)$. Conclusions Postoperative change of PROS length was similar in idiopathic and diabetic ERMs. PROS length change may not be an indicator of postoperative visual acuity. Eye (2015) 29, 1446-1452; doi:10.1038/eye.2015.133; published online 17 July 2015

\section{Introduction}

Epiretinal membranes (ERMs) are mainly classified as idiopathic and secondary. The growth of fibrocellular tissue on the inner limiting membrane (ILM) due to a break in it following posterior vitreous detachment (PVD) is the pathogenesis of idiopathic ERMs. ${ }^{1}$
K Yüksel' ${ }^{1}$ Y Karaküçük², A Özkaya', G Pekel³, Ö Baz' ${ }^{1}$ C Alagöz ${ }^{1}$ and AT Yazıcı ${ }^{1}$
A secondary ERM has different properties from an idiopathic ERM, because several

inflammatory and vascular processes take role in its pathogenesis. ${ }^{2,3}$ Retinal vascular diseases, diabetes mellitus (DM), uveitis, ocular traumas, argon laser photocoagulation, retinal tears, and detachments are the most known etiologic factors of secondary ERMs. ${ }^{4}$ The standard treatment of ERMs is pars plana vitrectomy (PPV) with membrane peeling.

The most important method for diagnosis of ERMs has become optical coherence tomography (OCT) in the last decade. Spectraldomain (SD) OCT has the capability of providing detailed and high-resolution images of macular microstructure. ${ }^{5}$ It is possible to obtain thickness values of photoreceptor layer by means of SD OCT. The microstructure of the outer retina, especially the photoreceptor layer, may reflect visual acuity in several disorders such as diabetic macular edema, age-related macular degeneration and macular hole. ${ }^{6-8}$

Some patients have poor visual acuity following a successful ERM surgery. This poor outcome sometimes cannot be explained by well-known prognostic factors such as duration of symptoms and preoperative visual acuity. ${ }^{9}$ It was reported that the photoreceptor outer segment (PROS) status may be a prognostic factor in idiopathic ERM surgery. ${ }^{10}$ Idiopathic and secondary ERMs differ significantly in terms of macular morphology and visual acuity. ${ }^{11} \mathrm{We}$ hypothesized that PROS may be also a prognostic factor for secondary ERM cases (ie diabetic ERMs). In this study, our aim was to compare the PROS length in diabetic and idiopathic epiretinal membranes.

\section{Materials and methods}

In this retrospective case series, 52 eyes of 50 patients who underwent PPV due to idiopathic 
and diabetic ERM were included. This study adhered to the tenets of the Declaration of Helsinki and was approved by the institutional ethics committee.

\section{Study population}

This study consisted of two groups: the idiopathic ERM group and diabetic ERM group. All the patients underwent PPV with ERM and ILM removal. The patients had been followed up at least 6 months postoperatively. There were 27 eyes of 25 patients in the idiopathic ERM group, whereas 25 eyes of 25 patients in the diabetic ERM group. Patients who had macular hole, vitreomacular traction, retinal detachment, macular ischemia, retinal neovascularization, uveitis, retinal vascular occlusions, and other ocular disorders were excluded. Subjects who had high-grade cataract or history of cataract surgery were also excluded. Low-quality OCT measurements were not included in the analysis. At each follow-up visit, all patients received an ophthalmic examination consisting of a visual acuity assessment (Snellen chart), biomicroscopy, Goldmann applanation tonometer, indirect retinoscopy, and macular OCT measurements. All preoperative examinations were repeated postoperatively at the first, third, and sixth months.

\section{Surgical technique}

Surgery consisted of 23-gauge transconjunctival sutureless PPV, ERM, and ILM peeling using a membrane forceps. Triamcinolone acetonide $(5.7 \mathrm{mg} / \mathrm{ml} 0.1-0.3 \mathrm{ml})$ was used to stain ERM. Brilliant blue was used to stain ILM. Infusion fluid or air was used for internal tamponade. In patients with a visually significant cataract, phacoemulsification with an intraocular lens implantation was performed before the PPV (8 eyes in the idiopathic ERM group and 16 eyes in the diabetic ERM group). Topical antibiotic (moxifloxacin) drops were prescribed five times a day for 2 weeks postoperatively. Topical steroid (prednisolone) drops were prescribed six times a day for 1 month and tapered off slowly. No intraoperative or postoperative complications were recorded related to surgery, as well as recurrent ERM formation.

\section{OCT measurements}

SD OCT (Spectralis, Heidelberg, Germany) was used in order to measure PROS length, overall PR length and central foveal thickness (CFT). This OCT provides 40000 A-scans per second, with a resolution of $7-14 \mu \mathrm{m}$ by using a 870-nm wavelength superluminescent diode. Spectralis has active eye tracking system that minimizes motion artifact. The PROS length was taken as the distance from inner surface of IS/OS (inner segment/outer segment) band to inner surface of retinal pigment epithelium (RPE). The overall PR length was taken as the distance from the outer border of external limiting membrane (ELM) to inner surface of RPE. The PROS and overall PR lengths were measured at two points: $500 \mu \mathrm{m}$ nasal and temporal to the center of fovea (Figure 1) and the average of two measurements were used for analysis. The measurements were performed by using manual caliber tools provided by the Spectralis device software. The images were analyzed with a zoom of $400 \%$. The evaluation of IS/OS integrity was a binary decision 'yes/no' in the 1-mm diameter circular area of the central fovea by examining multiple scans. All measurements were made by two experienced researchers (KY, GP). The OCT examinations were repeated at least three times, and the best-quality performances were selected for analysis. For quantitative data on PROS and overall PR length, the average values obtained by the two investigators were used. The OCT images were evaluated also to determine the integrity of the IS/OS junction and type of macular thickening (ie diffuse or cystoid).

\section{Statistical analysis}

For statistical analysis, Statistical Package for the Social Sciences version 16.0 software for Windows (SPSS Inc., Chicago, IL, USA) was used. All data are expressed as the mean \pm SD of the mean. $P$-values $<0.05$ were considered to be statistically significant. Snellen chart readings were converted to logMAR for statistical analysis. Friedman's test was applied for each outcome measures (ie, PROS length, overall PR length, and CFT) within each study group. Post hoc analysis with Wilcoxon signed-rank tests was conducted with a Bonferroni correction applied, resulting in a significance level set at $P<0.0125$. We applied Mann-Whitney $U$-test to test the differences between the parameters in the idiopathic and diabetic ERM groups. Spearman correlation analysis was used to detect association between PROS length change, CFT decrease, and visual acuity gain in both groups. The $\chi^{2}$-test was used to determine whether there was a significant difference between the observed frequencies of IS/OS junction disruption in the two study groups.

\section{Results}

The mean age of the patients in the idiopathic ERM group was $69.1 \pm 5.9$ years (range: $59-80$ years), and the mean age of the participants in the diabetic ERM group was $67.0 \pm 7.2$ years (range: $53-84$ yearsp; $P=0.24$ ). There were 12 male and 13 female patients in both groups $(P=1.00)$. There were 14 right eyes (52\%) and 13 left eyes (48\%) in 


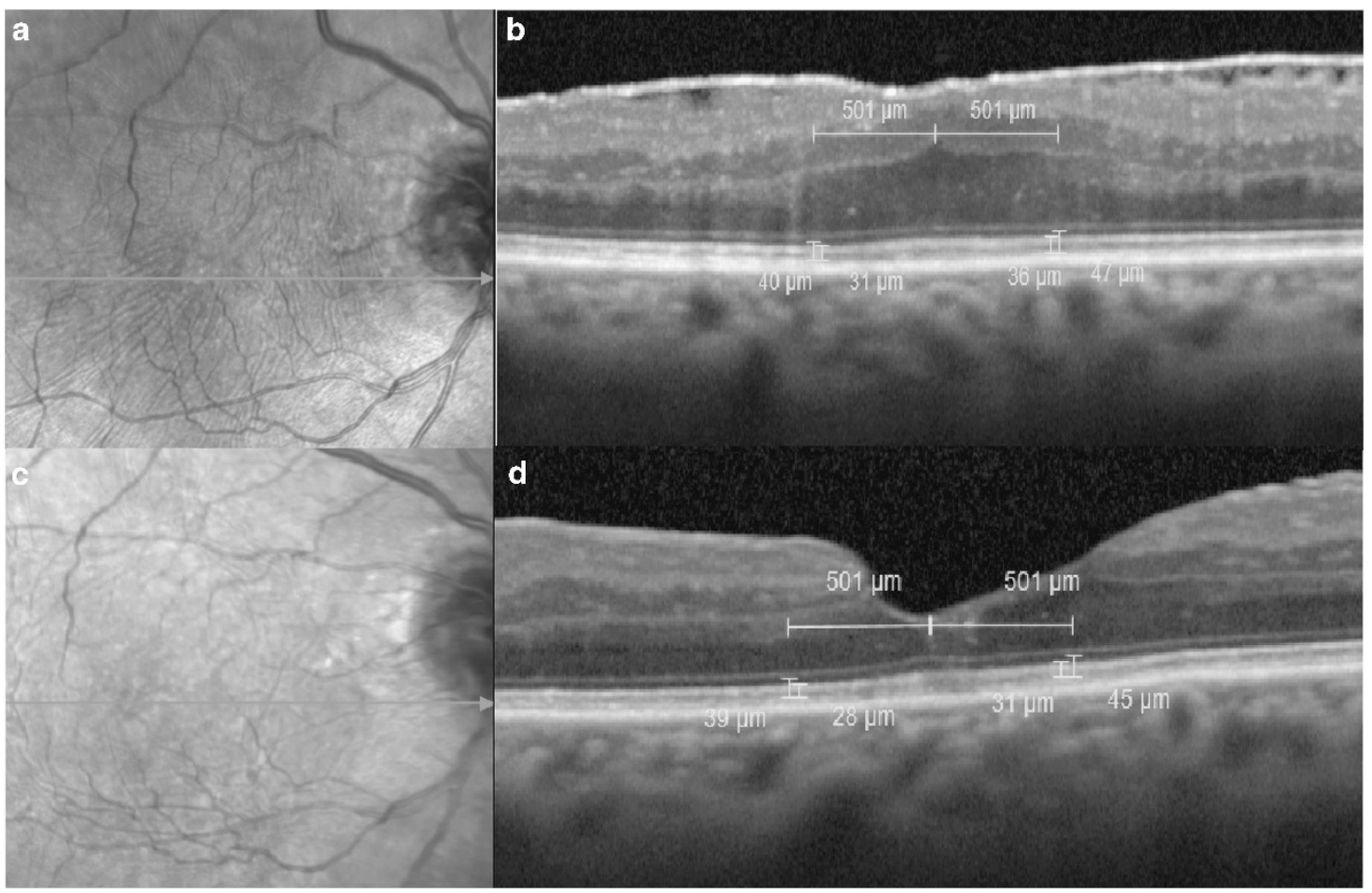

Figure 1 Spectral domain OCT images that show the preoperative (b) and postoperative (d) measurements of PROS and overall PR length, as well as the posterior segment photos (a and c).

Table 1 The mean PROS, overall PR thickness, and CFT values of the 'idiopathic' ERM group before and after surgery are presented

\begin{tabular}{lcccc}
\hline & Pre-op. & 1st month & 3rd month & 6th month \\
\hline PROS $(\mu \mathrm{m})$ & $33.0 \pm 4.7$ & $30.1 \pm 3.9(P<0.001)^{\mathrm{a}}$ & $29.6 \pm 4.1(P<0.001)^{\mathrm{a}}$ & $28.6 \pm 4.3(P<0.001)^{\mathrm{a}}$ \\
Overall PR $(\mu \mathrm{m})$ & $44.1 \pm 4.7$ & $40.1 \pm 3.9(P<0.001)^{\mathrm{a}}$ & $39.2 \pm 4.0(P<0.001)^{\mathrm{a}}$ & $38.9 \pm 3.8(P<0.001)^{\mathrm{a}}$ \\
CFT $(\mu \mathrm{m})$ & $477.0 \pm 92.7$ & $414.4 \pm 63.8(P<0.001)^{\mathrm{a}}$ & $371.2 \pm 86.7(P<0.001)^{\mathrm{a}}$ & $352.8 \pm 53.4(P<0.001)^{\mathrm{a}}$ \\
\hline
\end{tabular}

Abbreviations: CFT, central foveal thickness; ERM, epiretinal membrane; PR, photoreceptor; PROS, photoreceptor outer segment. ${ }^{a} P$-value is calculated according to the comparison to the preoperative measurements.

the idiopathic group, whereas there were 9 right eyes $(36 \%)$ and 16 left eyes $(64 \%)$ in the diabetic group.

The IS/OS junction disruption was found in 11 eyes ( $41 \%)$ in the idiopathic ERM group, whereas it was found in 18 eyes (72\%) in the diabetic ERM group preoperatively $(P=0.02)$. In the idiopathic ERM group, the type of preoperative macular thickening was diffused in 23 eyes $(85 \%)$ and cystoid in 4 eyes (15\%). In the diabetic ERM group, the type of preoperative macular thickening was diffuse in 6 eyes (\%24), and cystoid in 19 eyes $(76 \%)$. There were no significant differences between the reduction in mean PROS $(P=0.29)$, overall PR length $(P=0.67)$, and CFT $(P=0.61)$ measurements and the presence of cystoid macular edema in the diabetic ERM group at the last visit.

The mean visual acuity values (logMAR) of the idiopathic ERM group in the preoperative, postoperative first, third, and sixth month were $0.50 \pm 0.20,0.33 \pm 0.16$, $0.26 \pm 0.15$, and $0.22 \pm 0.16$, respectively $(P<0.001$ for all the postoperative values when compared with preoperative visual acuity). The mean visual acuity values ( $\log$ MAR) of the diabetic ERM group in the preoperative, postoperative first, third, and sixth month were $0.91 \pm 0.36,0.68 \pm 0.35,0.63 \pm 0.38$, and $0.66 \pm 0.38$, respectively $(P<0.001$ for all the postoperative values when compared with preoperative visual acuity). The visual acuity value of the idiopathic ERM group was significantly higher than that of diabetic ERM group in all the visits $(P<0.001)$. Preoperative visual acuity was statistically significantly low in eyes with IS/OS junction disruption in both the idiopathic ERM $(P<0.001)$ and diabetic ERM $(P<0.001)$ groups.

The mean PROS, overall PR length and CFT measurements of the idiopathic and diabetic ERM groups 
Table 2 The mean PROS, overall PR thickness and CFT values of the 'diabetic' ERM group before and after surgery are presented

\begin{tabular}{lcccr}
\hline & Pre-op. & 1st month & 3rd month & 6th month \\
\hline PROS $(\mu \mathrm{m})$ & $32.2 \pm 3.0$ & $28.9 \pm 2.4(P<0.001)^{\mathrm{a}}$ & $28.7 \pm 2.4(P<0.001)^{\mathrm{a}}$ & $28.8 \pm 2.3(P<0.001)^{\mathrm{a}}$ \\
Overall PR $(\mu \mathrm{m})$ & $42.4 \pm 3.2$ & $38.3 \pm 2.4(P<0.001)^{\mathrm{a}}$ & $37.8 \pm 2.6(P<0.001)^{\mathrm{a}}$ & $37.9 \pm 2.5(P<0.001)^{\mathrm{a}}$ \\
CFT $(\mu \mathrm{m})$ & $543.1 \pm 107.9$ & $415.6 \pm 98.6(P<0.001)^{\mathrm{a}}$ & $347.9 \pm 96.4(P<0.001)^{\mathrm{a}}$ & $317.0 \pm 82.8(P<0.001)^{\mathrm{a}}$ \\
\hline
\end{tabular}

Abbreviations: CFT, central foveal thickness; ERM, epiretinal membrane; Pre-op., preoperative; PR, photoreceptor; PROS, photoreceptor outer segment.

${ }^{a} P$-value is calculated according to the comparison to the preoperative measurements.

Table 3 The amounts of decrease in the measurements of PROS length, overall PR length, and CFT at first month, third month, and sixth month postoperative visits in the idiopathic and diabetic ERM groups are presented

\begin{tabular}{lccr}
\hline & $\begin{array}{c}\text { Idiopathic } \\
\text { ERM group }\end{array}$ & $\begin{array}{c}\text { Diabetic } \\
\text { ERM group }\end{array}$ & P-value \\
\hline PROS length $(\mu \mathrm{m})$ & & & \\
1st month & $2.9 \pm 2.1$ & $3.3 \pm 2.3$ & 0.10 \\
3rd month & $3.4 \pm 3.2$ & $3.5 \pm 2.6$ & 0.40 \\
6th month & $4.4 \pm 2.5$ & $3.4 \pm 2.8$ & 0.38 \\
& & & \\
Overall PR length $(\mu \mathrm{m})$ & & \\
1st month & $4.0 \pm 2.7$ & $4.1 \pm 2.5$ & 0.40 \\
3rd month & $4.9 \pm 4.3$ & $4.7 \pm 2.6$ & 0.19 \\
6th month & $5.2 \pm 3.2$ & $4.5 \pm 2.5$ & 0.89 \\
& & & \\
CFT ( $\mu$ m) & & & 0.006 \\
1st month & $62.6 \pm 58.9$ & $127.5 \pm 86.9$ & 0.004 \\
3rd month & $105.8 \pm 115.6$ & $195.2 \pm 110.1$ & $<0.001$ \\
6th month & $124.2 \pm 81.8$ & $226.4 \pm 110.5$ & $<$ \\
\hline
\end{tabular}

Abbreviations: CFT, central foveal thickness; ERM, epiretinal membrane; PR, photoreceptor; PROS, photoreceptor outer segment.

are presented in Table 1 and Table 2, respectively. All the studied parameters were statistically significantly lower at all the postoperative visits when compared with preoperative values in both groups $(P<0.001)$.

Preoperative mean CFT was higher in the diabetic ERM group $(P=0.03)$, whereas preoperative PROS and overall PR length measurements were similar in the two study groups ( $P=0.75$ and $P=0.25$, respectively). The amount of decrease in PROS and overall PR length were similar in both groups after surgery $(P>0.05)$. Table 3 shows the amounts of decrease in the measurements of PROS length, overall PR length, and CFT at first, third, and sixth month postoperative visits in the idiopathic and diabetic ERM groups.

The scatter plot graphics that show the correlation of visual acuity difference and PROS length difference between the preoperative and last visits are presented in Figure 2. Visual acuity gain was correlated with CFT decrease in the diabetic ERM group (rho $=0.41, P=0.03$ ), while there was no correlation in the idiopathic ERM group (rho $=0.09, P=0.66$ ) at the last postoperative visit.

\section{Discussion}

The present study showed that removal of idiopathic and diabetic ERMs both resulted in postoperative decreased PROS and overall PR length in similar amounts. Our results also suggest that the correlation of visual acuity gain and PROS length difference between the preoperative and last visits is not high in both groups. However, the two groups showed different characteristics in the aspect of IS/OS junction disruption, visual acuity, and macular thickening.

Visual acuity is usually found to be worse in secondary ERMs such as diabetic ERMs when compared with idiopathic ERMs. ${ }^{11}$ Our study also showed that mean visual acuity values were lower in the diabetic ERMs both preoperatively and postoperatively. Nevertheless both groups showed significant visual acuity gain postoperatively. Visual acuity was found to be strongly associated with IS/OS junction disruption in ERM cases. ${ }^{12,13}$ In the diabetic ERM group, IS/OS junction disruption was more frequent which explains the low visual acuity in this group.

The PROS length is considered as an indicator of visual acuity at all follow-up visits after surgery and a predictor of postoperative visual acuity in patients with idiopathic ERM. ${ }^{14}$ Our results showed that visual acuity increased, whereas PROS length decreased postoperatively; however, there was no significant association between amount of PROS length decrease and visual acuity increase. This outcome may be explained by StilesCrawford effect that points out the directional sensitivity of the cone receptors. ${ }^{15-17}$ The visual system has reduced sensitivity to light rays that are not concordant with the direction of the photoreceptors. ${ }^{15-17}$ It was reported that when the integrity and orientation of the photoreceptors is disturbed by edema or traction, directional light sensitivity of the cones decline. ${ }^{16,18}$ According to us, since the foveal architecture was improved postoperatively, the directional sensitivity of the photoreceptors increased, and the visual acuity gain occurred in the present study.

Shin et $a l^{19}$ found that the mean macular PROS length was $\sim 37 \mu \mathrm{m}$ in patients with ERM. In our study, the mean preoperative PROS length was $33 \mu \mathrm{m}$ in the idiopathic ERMs, whereas it was $32 \mu \mathrm{m}$ in the in the diabetic ERMs. 

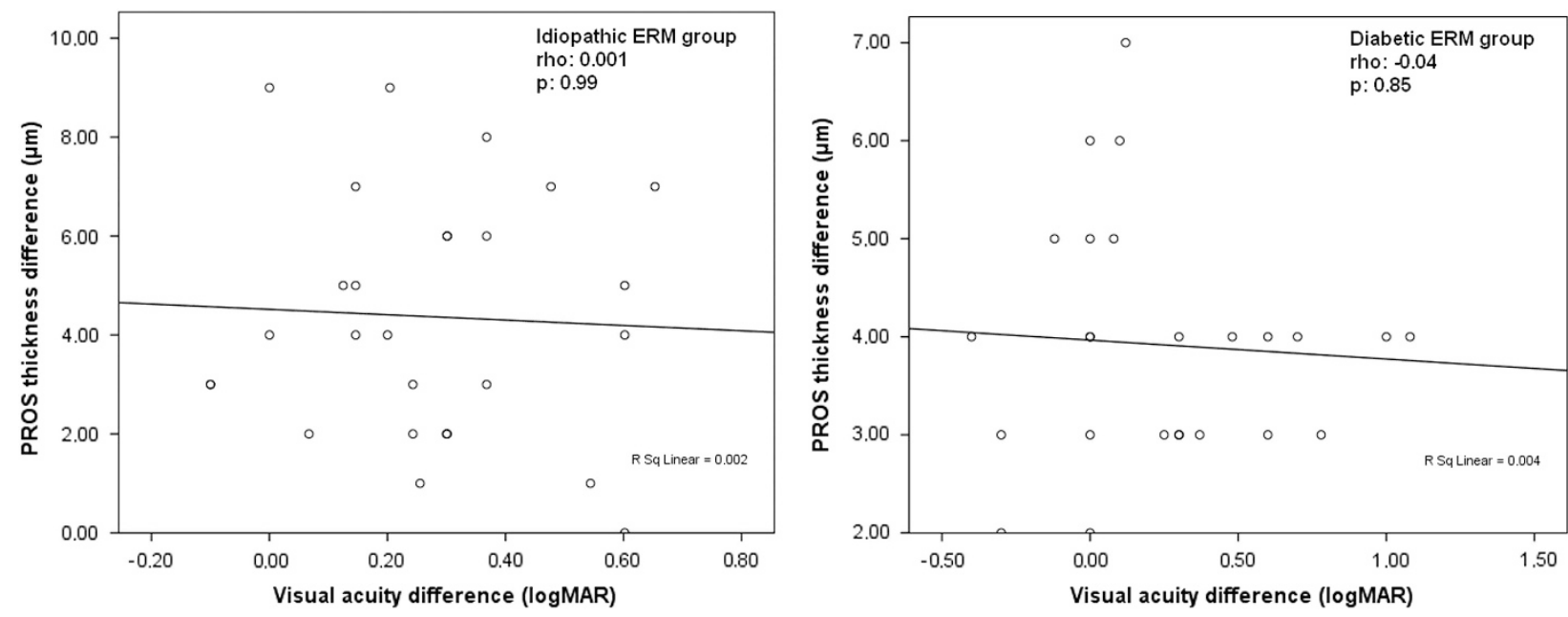

Figure 2 Scatter plot graphics that show the correlation of visual acuity gain and PROS length difference between the preoperative and last visits in diabetic and idiopathic ERM groups.

The PROS and overall PR length decreased significantly in both groups at all follow-up visits in the present study. Postoperative decrease in PROS length might be due to the relief of upward mechanical traction of ERM. Shiono et al $^{14}$ reported that PROS length and outer foveal thickness were significantly decreased 1 month after surgery compared with that at baseline, however, they returned to baseline values at 3 and 6 months after surgery in idiopathic ERMs. The difference in the change of postoperative PROS length at third and sixth month visits between our study and Shiono's study may be occurred due to the longer symptomatic period until surgery in our patients.

There are some other reports related to the status of photoreceptor layer in ERM. ${ }^{20-22} \mathrm{Kim}$ et al reported that the preoperative integrity of foveal photoreceptors may be an important prognostic factor for visual outcome in patients with idiopathic ERM. ${ }^{20}$ Itoh et $a l^{21}$ found that the recovery of the foveal photoreceptor cone outer segment tips line disintegrity is associated with the visual recovery after ERM surgery. In addition, the presence of a preoperative intact IS/OS junction was found to be an important predictor of better visual outcome following ERM surgery. ${ }^{22}$

The extent of macular thickness and presence of cystoid macular edema are some of the factors that may affect the functional and anatomical success of the ERM removal. ${ }^{12,23}$ Yazici et al reported that idiopathic and diabetic ERMs differ significantly in terms of macular thickness and presence of cystoid macular edema. ${ }^{11}$ In our study, eyes with diabetic ERMs had much more macular cystoid spaces and had thicker central fovea compared to eyes with idiopathic ERMs. However, the central macular thickness decreased significantly in a similar manner in both the diabetic and idiopathic ERM groups following surgery. Romano et al ${ }^{24}$ reported that large intraretinal cysts may induce subfoveal atrophy after vitrectomy with ILM peeling for diabetic macular edema. In our study, there were no significant differences between the reduction in mean PROS, overall PR length, and CFT measurements and the presence of cystoid macular edema in the diabetic ERMs.

ILM removal in ERM surgery is a controversial issue. Ahn $e t a l^{25}$ suggested that in terms of visual outcome and photoreceptor integrity, ILM peeling in ERM surgery may not have an additional benefit. Kang et $\mathrm{al}^{26}$ reported that removal of the ILM may lower recurrence rates in both idiopathic and secondary ERM cases. In our study, ILM peeling was performed in all the cases and we did not encounter recurrent ERM in both the diabetic and idiopathic groups. However, it was reported that ILM and ERM are not always distinct and surgically separable as previously recognized, and it may be difficult to determine the nature of membranes intraoperatively. ${ }^{27} \mathrm{As}$ an oppose finding for the benefit of ILM removal, Grimbert $e a^{28}$ reported that active ILM peeling may cause postoperative visual discomfort related to micro trauma.

Our study had several limitations. Measurements of PROS and overall PR length were performed manually using caliber tools of the software. A full-automated image analysis program for PROS length measurement would provide more accurate data. Retrospective study design and relatively small sample sizes are additional limitations of this study.

In conclusion, the amount of postoperative decrease of PROS and overall PR length are similar in idiopathic and diabetic ERMs. Diabetic ERMs differ from idiopathic 
ERMs in terms of visual acuity at each visit, preoperative presence of cystoid spaces and IS/OS disruption. We should suggest that PROS length change may not be an important determinant of postoperative visual acuity gain following surgery for ERM removal in both idiopathic and diabetic ERMs.

\section{Summary}

What was known before

- The photoreceptor outer segment (PROS) length may be a prognostic factor in idiopathic epiretinal membrane (ERM) surgery.

What this study adds

- Postoperative change of PROS length was similar in idiopathic and diabetic ERMs.

- PROS length change may not be an indicator of postoperative visual acuity.

\section{Conflict of interest}

The authors declare no conflicts of interest.

\section{References}

1 Joshi M, Agrawal S, Christoforidis JB. Inflammatory mechanisms of idiopathic epiretinal membrane formation. Mediators Inflamm 2013; 2013: 192582.

2 Hiscott PS, Grierson I, McLeod D. Natural history of fibrocellular epiretinal membranes: a quantitative, autoradiographic, and immunohistochemical study. $\mathrm{Br} J$ Ophthalmol 1985; 69: 810-823.

3 Harada C, Mitamura Y, Harada T. The role of cytokines and trophic factors in epiretinal membranes: involvement of signal transduction in glial cells. Prog Retin Eye Res 2006; 25 149-164.

4 Appiah AP, Hirose T. Secondary causes of premacular fibrosis. Ophthalmology 1989; 96: 389-392.

5 Srinivasan VJ, Wojtkowski M, Witkin AJ, Duker JS, Ko TH, Carvalho $\mathrm{M}$ et al. High-definition and 3-dimensional imaging of macular pathologies with high speed ultrahighresolution optical coherence tomography. Ophthalmology 2006; 113: 2054.e1-2054.14.

6 Forooghian F, Stetson PF, Meyer SA, Chew EY, Wong WT, Cukras C et al. Relationship between photoreceptor outer segment length and visual acuity in diabetic macular edema. Retina 2010; 30: 63-70.

7 Pappuru RR, Ouyang Y, Nittala MG, Hemmati HD, Keane PA, Walsh AC et al. Relationship between outer retinal thickness substructures and visual acuity in eyes with dry age-related macular degeneration. Invest Ophthalmol Vis Sci 2011; 52: 6743-6748.

8 Shimozono M, Oishi A, Hata M, Kurimoto Y. Restoration of the photoreceptor outer segment and visual outcomes after macular hole closure: spectral-domain optical coherence tomography analysis. Graefes Arch Clin Exp Ophthalmol 2011; 249: 1469-1476.

9 Asaria R, Garnham L, Gregor ZJ, Sloper JJ. A prospective study of binocular visual function before and after successful surgery to remove a unilateral epiretinal membrane. Ophthalmology 2008; 115: 1930-1937.

10 Shimozono M, Oishi A, Hata M, Matsuki T, Ito S, Ishida K et al. The significance of cone outer segment tips as a prognostic factor in epiretinal membrane surgery. Am J Ophthalmol 2012; 153: 698-704.

11 Yazici AT, Alagöz N, Celik HU, Bozkurt E, Alagöz C, Cakir $\mathrm{M}$ et al. Idiopathic and secondary epiretinal membranes: do they differ in terms of morphology? An optical coherence tomography-based study. Retina 2011; 31: 779-784.

12 Suh MH, Seo JM, Park KH, Yu HG. Associations between macular findings by optical coherence tomography and visual outcomes after epiretinal membrane removal. Am J Ophthalmol 2009; 147: 473-480.

13 Inoue M, Morita S, Watanabe Y, Kaneko T, Yamane S, Kobayashi $\mathrm{S}$ et al. Inner segment/outer segment junction assessed by spectral-domain optical coherence tomography in patients with idiopathic epiretinal membrane. Am J Ophthalmol 2010; 150: 834-839.

14 Shiono A, Kogo J, Klose G, Takeda H, Ueno H, Tokuda N et al. Photoreceptor outer segment length: a prognostic factor for idiopathic epiretinal membrane surgery. Ophthalmology 2013; 120: 788-794.

15 Gao W, Cense B, Zhang Y, Jonnal RS, Miller DT. Measuring retinal contributions to the optical Stiles-Crawford effect with optical coherence tomography. Opt Express 2008; 16: 6486-6501.

16 Zagers NP, Pot MC, van Norren D. Spectral and directional reflectance of the fovea in diabetes mellitus: photoreceptor integrity, macular pigment and lens. Vision Res 2005; 45: 1745-1753.

17 DeLint PJ, Vos JJ, Berendschot TT, van Norren D. On the Stiles-Crawford effect with age. Invest Ophthalmol Vis Sci 1997; 38: 1271-1274

18 Lardenoye CW, Probst K, DeLint PJ, Rothova A. Photoreceptor function in eyes with macular edema. Invest Ophthalmol Vis Sci 2000; 41: 4048-4053.

19 Shin YU, Cho HY, Lee BR. Outer photoreceptor layer thickness mapping in normal eyes and eyes with various macular diseases using spectral domain optical coherence tomography: a pilot study. Graefes Arch Clin Exp Ophthalmol 2013; 251: 2529-2537.

20 Kim HJ, Kang JW, Chung H, Kim HC. Correlation of foveal photoreceptor integrity with visual outcome in idiopathic epiretinal membrane. Curr Eye Res 2014; 39: 626-633.

21 Itoh Y, Inoue M, Rii T, Hirota K, Hirakata A. Correlation between foveal cone outer segment tips line and visual recovery after epiretinal membrane surgery. Invest Ophthalmol Vis Sci 2013; 54: 7302-7308.

22 Cobos E, Arias L, Ruiz-Moreno J, Rubio M, Garcia-Bru P, Caminal J et al. Preoperative study of the inner segment/ outer segment junction of photoreceptors by spectraldomain optical coherence tomography as a prognostic factor in patients with epiretinal membranes. Clin Ophthalmol 2013; 7: 1467-1470.

23 Wilkins JR, Puliafito CA, Hee MR, Duker JS, Reichel E, Coker JG et al. Characterization of epiretinal membranes using optical coherence tomography. Ophthalmology 1996; 103: 2142-2151.

24 Romano MR, Romano V, Vallejo-Garcia JL, Vinciguerra R, Romano M, Cereda $\mathrm{M}$ et al. Macular hypotrophy after internal limiting membrane removal for diabetic macular edema. Retina 2014; 34: 1182-1189. 
25 Ahn SJ, Ahn J, Woo SJ, Park KH. Photoreceptor change and visual outcome after idiopathic epiretinal membrane removal with or without additional internal limiting membrane peeling. Retina 2014; 34: 172-181.

26 Kang KT, Kim KS, Kim YC. Surgical results of idiopathic and secondary epiretinal membrane. Int Ophthalmol 2014; 34: $1227-1232$.
27 DeMarchis EH, Pershing S, Moshfeghi DM. Clinicalpathologic correlation: vitrectomy with epiretinal and internal limiting membrane peel. Ophthalmic Surg Lasers Imaging Retina 2014; 45: 218-221.

28 Grimbert P, Lebreton O, Weber M. Optical coherence tomography and microperimetry after internal limiting membrane peeling forepiretinal membrane. J Fr Ophtalmol 2014; 37: 434-441. 